\title{
Does Opioid Use for Pain Management Warrant Routine Bone Mass Density Screening in Men?
}

Joseph D. Fortin, DO', Gina M. Bailey, BS², and Joel A. Vilensky, PhD²

From: ${ }^{1}$ Spine Technology and Rehabilitation, Fort Wayne, IN; ${ }^{2}$ Department of Anatomy and Cell Biology, Indiana University School of Medicine, Fort Wayne, IN

Address correspondence: Mrs. Gina M. Bailey Indiana University School of Medicine

2101 E. Coliseum Blvd.

Fort Wayne, IN 46805

Email: gbailey6@comcast.net

Disclaimer: There was no external funding in the preparation of this manuscript.

Conflict of interest: None.

Manuscript received: 02/21/2008 Revised manuscript received: 04/30/2008

Accepted for publication: 06/10/2008

Free full manuscript: www.painphysicianjournal.com
Background: Although opioids are known to inhibit testosterone production and bone formation, no prior study has investigated the relationship between opioid use in male subjects and bone mass density measurements.

Method: Eighty-one male patients who had been using opioids for a period of time from a few weeks to 20 years were included in this study. Blood samples were obtained to examine the total testosterone level of these subjects and each patient also had a bone mass density scan. This study was done retrospectively using lab values collected during the normal care of the patient. No extra measures were done relative to this study and all patient identifiers were removed from the data prior to analysis. Accordingly, there was no need here for an IRB or patient consent.

Results: Fifty percent of hypogonadal men had bone mass densities in the osteoporotic or osteopenic range. Forty-two percent of the men who had total testosterone levels within the normal range also had bone mass densities within these ranges. Other factors may also influence the bone mass density results. For example, in this study $52 \%$ of the patients disclosed that they smoke.

Conclusion: Of this population almost half of the men had bone mass densities in the osteopenic/osteoporotic ranges, regardless of their total testosterone blood level. Therefore, monitoring the total testosterone blood level is not a reliable method to determine the risk for developing opioid-associated osteoporosis. Further investigations should be done to help evaluate whether routine bone mass density screenings for men prescribed opioids for chronic pain management may be warranted.

Key words: Opioid, OPIAD, hypogonadal, bone mass density

Pain Physician 2008; 11:4:539-541

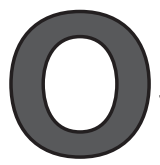

pioid use is a newly recognized risk factor for the development of osteoporosis in opioidconsuming young adults (1). Such patients have been reported to have a higher incidence of hip fractures than controls (1).

Opioid use may result in opioid-induced androgen deficiency (OPIAD) $(1,2)$. Specifically, testosterone synthesis and hypothalamic gonadotrophin releasing hormone are inhibited by opioids $(1,3)$. It is estimated that in the US as many as 5 million men with chron- ic non-malignant pain have OPIAD (4), which is associated with decreased libido, erectile dysfunction, fatigue, depressed moods, and hot flashes (1). The long-term effects of low testosterone may result in anemia, sarcopenia, and osteoporosis $(1,4)$. Daniell (2) showed that opiate-induced decreases in testosterone levels occasionally approached the castrate range and were associated with weakness and diminished muscle mass, which presumably contributed to an increased fall and fracture risk. 
Opioids also interfere with bone formation independently of hormone changes $(1,2)$. There is a decrease in serum osteocalcin levels in patients who use opioids (1). A large concentration of opioid receptors in osteoblasts inhibits the formation of osteocalcin $(1,2)$.

The purpose of this study was to examine the bone mass density of male patients who have been prescribed opioids for pain management. Furthermore, we sought to determine if a correlation of the total testosterone levels of patients using opioids and bone mass density exists, or if bone mass density is compromised independently of the testosterone level.

\section{Methods and Materials}

Eighty-one male patients ( 20 to 84 years old) who sought treatment from a regional pain management clinic for chronic pain were included in the study. These patients used opioids to control their pain from a few weeks to 20 years. Total testosterone levels were obtained from blood and bone mass density scans were performed on a recently calibrated Hologic QDR-1000 DXA scanner for all the patients.

Hypogonadism was defined as a total serum testosterone level less than $280 \mathrm{ng} / \mathrm{dL}$. World Health Organization (WHO) criteria for osteopenia and osteoporosis were used. A T-score -1 to -2.5 standard deviations below the mean indicates osteopenia, and a T-score below -2.5 standard deviations indicates osteoporosis. These values are based on post-menopausal Caucasian women. Non-Caucasian female and male standard have not been established at this time.

\section{Results}

The average age of the 81 patients was 45 years. The average duration on opioids was 2.5 years. Thirty-six patients (44\%) had bone mass densities in the osteopenic and osteoporotic ranges (Table 1). Twentytwo patients $(27 \%)$ included in this study were con- sidered hypogonadal (Table 1). Eleven of the patients $(50 \%)$ that were hypogonadal had bone mass densities in the osteopenic or osteoporotic range (Table 1). More interestingly, 25 men (42\%) had a total testosterone level within normal range and were osteopenic or osteoporotic (Table 1).

\section{Discussion}

We found that $44 \%$ of the men involved in this study had bone mass densities in the osteopenic/osteoporotic range, even though only $27 \%$ were hypogonadal. Forty-two percent of the patients whose testosterone values were within normal limits also had lowered bone mass densities. Fifty-three percent of the men in the study disclosed that they smoked.

The reference ranges for osteoporosis and osteopenia are based on the WHO criteria of a population of post-menopausal Caucasian women. The reference range for men has not been established at this time. Therefore, the osteopenic/osteoporotic classification of our subjects is approximate but undoubtedly underestimates the percentage in men, because of their higher bone density values.

The production of testosterone decreases naturally as men age (5). The term andropause has been used to describe the phenomena of approximately $1 \%$ decrease of testosterone per year decline after the age of 30 in men (5). It is also believed that hypogonadism is better determined by the symptoms than by a specific serum level because testosterone levels causing dysfunction vary widely among individuals (5). Hypogonadal symptoms may be present in patients who have a total testosterone level within normal limits. Therefore, even the patients who have normal-appearing testosterone levels may exhibit the effects of hypogonadism, including decreased bone mass density.

Additional factors may also contribute to a decrease in bone mass density. Lifestyle choices such as smoking, alcohol intake, drugs, and other existing

Table 1. Bone Density Results

\begin{tabular}{|l|c|c|c|c||}
\hline Testosterone Range & Normal & Osteopenic & Osteoporotic & Total \\
\hline Hypogonadal & $11(50 \%)$ & $9(41 \%)$ & $2(9 \%)$ & $22(27 \%)$ \\
\hline Non-hypogonadal & $34(58 \%)$ & $20(34 \%)$ & $5(8 \%)$ & $59(73 \%)$ \\
\hline Total & $\mathbf{4 5 ( 5 6 \% )}$ & $\mathbf{2 9 ( 3 6 \% )}$ & $\mathbf{7 ( 8 \% )}$ & $\mathbf{8 1}(\mathbf{1 0 0 \% )}$ \\
\hline
\end{tabular}


medical conditions are all risk factors for developing osteoporosis (6). Scartberg et al (7) showed that total and free testosterone levels were positively and significantly associated with tobacco consumption, and that smokers have significantly higher levels of total testosterone. They also showed that men with a high body mass index (BMI) were more likely and smokers less likely to be in the lowest quintile of total testosterone (7). Vermeulen et al (8) showed that in healthy men the BMI accentuated the decline in androgen levels but may be partially masked when data are not corrected for the influence of smoking. Fiftythree percent of the participants in this study smoked. Forty-nine percent of the smokers had bone density values in the osteopenic/osteoporotic ranges. Alcohol consumption, BMI, and activity levels were not evaluated, but could possibly contribute to the reduced bone mass density of our patients.

Opioid use may induce hypogonadism $(1,2)$. The National Institutes of Health in 2002 recommended that physicians pay particular attention to the skeletal health in individuals with certain medical conditions or who are taking certain drugs (9). They included persons with hypogonadism in this group.
This study does have some limitations. The patients in this study were referral patients. As many pain intervention practices are well aware, these patients have already been treated with opioids prior to seeking care from their practices. Therefore, baseline data for the DEXA scan and testosterone levels were unavailable. Other variables historically affecting bone density, such as medication, nutrition, weight bearing activity, etc., may also impact the bone density data.

The total testosterone level may not a reliable screen for determining hypogonadism in men using opioids. Bone density screening is the only definitive and practical means to monitor the bone mass density in these patients. Our findings for the population of this study suggest that further investigations should be undertaken in an effort to help determine whether patients on opioid therapy for one year or more should have a DEXA scan to monitor bone mass density.

\section{Conclusion}

Routine screening for bone mass density may be an excellent asset in the management of men prescribed opioids for chronic pain, because it may prevent additional health problems associated with osteoporosis.

\section{References}

1. Daniell HW. Opioid-induced androgen deficiency. Curr Opin Endocrinol Diabetes 2006; 13:262-266.

2. Daniell HW. Opioid osteoporosis. Arch Intern Med 2004; 164:338.

3. Daniell HW. Hypogonadism in men consuming sustained-action oral opioids. J Pain 2002; 3:377-384.

4. Daniell HW, Lentz R, Mazer N. Open-label pilot study of testosterone patch therapy in men with opioid-induced androgen deficiency. J Pain 2006; 7:200-210.

5. Lewis B, Legato M, Fisch H. Medical implications of the male biological clock. JAMA 2006; 296:2369-2371.

6. Poole K, Compston J. Osteoporosis and its management. $B M J$ 2006; 333:12511256.

7. Svartberg J, Midtby M, Bonaa K, Sundsfjord J, Joakimsen R, Jorde R. The associations of age, lifestyle factors and chronic disease with testosterone in men: The Tromso study. Eur J Endocrinol 2003; 149:145-152.

8. Vermeulen A, Kaufman JM, Giagulli VA. Influence of some biological indexes on sex hormone-binding globulin and androgen levels in aging or obese males. J Clin Endocrinol and Metab 1996; 81: 1821-1826.

9. Marwick C. Consensus panel considers osteoporosis. JAMA 2000; 283:20932095. 